\title{
Characterization of polyvinyl alcohol-borax/ agarose (PVA-B/AG) double network hydrogel utilized for the cleaning of works of art
}

\author{
Ehab Al-Emam ${ }^{1,2^{*}}$, Hilde Soenen ${ }^{3}$, Joost Caen ${ }^{4}$ and Koen Janssens ${ }^{2}$
}

\begin{abstract}
Since cleaning of artworks may cause undesirable physicochemical alterations and is a nonreversible procedure, it is mandatory to adopt the proper cleaning procedure. Such a procedure should remove undesired materials whilst preserving the original surface. In this regard, numerous gels have been developed and exploited for the cleaning of various artwork surfaces. Lately, agarose (AG) and polyvinyl alcohol-borax (PVA-B) hydrogels have been widely employed as cleaning tools by conservators. Both hydrogels show some limitations in specific cleaning practices. In this work, we investigated the influence of including increased levels of agarose into PVA-B systems. For this reason, we performed a detailed characterization on the double network (DN) hydrogel including the chemical structure, the liquid phase retention, the rheological behavior, and the self-healing behavior of various PVA-B/AG double network hydrogels. These new hydrogels revealed better properties than PVA-B hydrogels and obviated their limitations. The inclusion of AG into PVA-B systems enhanced the liquid retention capacity, shape-stability, and mechanical strength of the blend. Furthermore, AG minimized the expelling/syneresis issue that occurs when loading PVA-B systems with low polarity solvents or chelating agents. The resultant double network hydrogel exhibits relevant self-healing properties. The PVA-B/AG double network is a new and useful cleaning tool that can be added to the conservators' tool-kit. It is ideal for cleaning procedures dealing with porous and complex structured surfaces, vertical surfaces and for long time applications.
\end{abstract}

Keywords: Cleaning, Conservation, Hydrogels, Polyvinyl alcohol-borax/agarose double network (PVA-B/AG DN), Rheology

\section{Introduction}

Cleaning of artworks is deemed to be one of the most important and critical actions undertaken by conservators owing to its irreversible nature. Cleaning processes include the removal of undesired deposits from the surface of the treated artwork for stability and aesthetical requirements $[1,2]$. In order to minimize the influence of the cleaning action on the underlying original materials, the cleaning action should be limited to the interface

\footnotetext{
*Correspondence: ehab.al-emam@uantwerpen.be; ehab_alemam@arch. sohag.edu.eg

1 Department of Conservation, Faculty of Archaeology, Sohag University, Sohag 82524, Egypt

Full list of author information is available at the end of the article
}

between the deposits and the surface to be treated. Using traditional poultices in combination with neat or blended solvents was the most common technique for surface cleaning. However, in some cleaning treatments, this approach proved to be inadequate as it may damage the original materials and/or leave residues behind [3-5]. To address these issues, so-called 'solvent-gels' were developed as a carrier for solvents and cleaning agents as a more controllable and selective cleaning tool [6]. Since then, conservation scientists have been exploring and developing variable types of gels to meet the different cleaning requirements [7-10]. In these studies, the gels were loaded with the desired cleaning agents and successfully employed to remove variable deposits from artwork surfaces.
Springer Open

(c) The Author(s) 2020. This article is licensed under a Creative Commons Attribution 4.0 International License, which permits use, sharing, adaptation, distribution and reproduction in any medium or format, as long as you give appropriate credit to the original author(s) and the source, provide a link to the Creative Commons licence, and indicate if changes were made. The images or other third party material in this article are included in the article's Creative Commons licence, unless indicated otherwise in a credit line to the material. If material is not included in the article's Creative Commons licence and your intended use is not permitted by statutory regulation or exceeds the permitted use, you will need to obtain permission directly from the copyright holder. To view a copy of this licence, visit http://creativeco mmons.org/licenses/by/4.0/. The Creative Commons Public Domain Dedication waiver (http://creativecommons.org/publicdomain/ zero/1.0/) applies to the data made available in this article, unless otherwise stated in a credit line to the data. 
In the last decade, agar and agarose gels were extensively exploited by conservators during cleaning of variable types of works of art such as: paper, stone, ceramic, textile, plaster, and wall paintings [11-16]. Agarose is a nonionic polysaccharide and is one of two components of agar gels. The other component is agaropectin which contains more sulphate groups than agarose and has a lower molecular weight. Agarose provides the gelling power for agar gels because of its high molecular weight. It is extracted from marine red seaweeds and is composed of $\beta-1,3$ linked-D-galactose, $\alpha-1$, 4-linked 3,6-anhydro-L-galactose (see Additional file 1: Fig. S1a). When agarose is heated in water above $85{ }^{\circ} \mathrm{C}$, random coils start to form. Upon cooling, the random coils transform into single and double helices in the initial gelation stage (Gel I). Further cooling leads to the final gelation stage where thick bundles of double helices are formed (Gel II) (see Additional file 1: Fig. S1b). This gelation process is thermoreversible and creates the 3D network of rigid agarose gel due to the formation of hydrogen bonds among the bundles of double helices $[1,17]$.

Despite of the advantage of those gels, allowing the inclusion of a broad range of cleaning agents [11, 18], one of their limitations is being (too) rigid. This lack of conforming to irregularly-shaped surfaces makes them more suitable for flat ones such as paper. This limitation may lead to uneven cleaning results due to the incomplete contact between the gel and the surface to be treated [19, 20]. To obviate this problem, the gel can be covered with glass plates to enhance the contact between the gel and the surface to be treated [11]. Another way to resolve this issue is by applying the gel by means of a brush in the sol-gel phase when it is still semi-solid. However, this approach can provoke detachment of fragile fragments from the treated surfaces as in the case of fragile paint layers. Moreover, since the gel is applied in the warm state $\left(c .40-45^{\circ} \mathrm{C}\right)$, it may cause damage to heat-sensitive materials [16].

On the other hand, polyvinyl alcohol-borax (PVAB) systems, which consist of PVA polymer crosslinked with borax, can serve as an alternative cleaning tool for conservators. PVA is characterized by a large number of hydroxyl group along its backbone. Inter- and intramolecular hydrogen bonds are formed among those hydroxyl groups. Thus, the number of hydroxyl group has a significant influence on PVA properties. In aqueous solutions of PVA, heat is required to improve the dissolution process especially in the case of PVA with a high degree of hydrolysis (containing more hydroxyl groups) $[21,22]$. Usually, sodium tetraborate decahydrate is used as a crosslinker for PVA-borax hydrogels. When it is dissolved in water, it dissociates to form equal amounts of boric acid $\left(\mathrm{B}(\mathrm{OH})_{3}\right), \mathrm{B}(\mathrm{OH})_{4}{ }^{-}$and $\mathrm{Na}^{+}$(Eq. 1) [23].

$$
\begin{aligned}
\mathrm{Na}_{2} \mathrm{~B}_{4} \mathrm{O}_{7} \cdot 10 \mathrm{H}_{2} \mathrm{O} \rightarrow & 2 \mathrm{~B}(\mathrm{OH})_{3}+2 \mathrm{~B}(\mathrm{OH})_{4}^{-} \\
& +2 \mathrm{Na}^{+}+3 \mathrm{H}_{2} \mathrm{O} .
\end{aligned}
$$

When a PVA solution is mixed with borax in the proper concentrations, the crosslinking process occurs in two steps: (a) the tetrahydroxy borate anion reacts with a diol of a PVA chain to form a monodiol complex; (b) the other two hydroxyl groups, attached to the boron atom in the borate, react with another adjacent diol. Thus, the crosslinking process is called didiol complexation (Additional file 1: Fig. S1c) [23, 24].

PVA-B systems are characterized by a viscoelastic behavior; as such, from a rheological point of view, they are not considered real gels. However, for simplicity, we will refer to them as hydrogels and this is also the term broadly used within the conservation community. The flexibility of these systems eases their conformity to surfaces with a rough morphology. Yet, they can be peeled off from the surface as one piece by the simple use of tweezers [25]. PVA-B systems can be loaded with organic solvents and chelators. The concentrations to be loaded depend on the hydrolysis degree of PVA polymer [24, 26]. Nonetheless, there are some limitations regarding the surfaces that PVA-B hydrogels can be applied to. It has been reported that some porous surfaces (e.g. some types of wood, paper, plaster) are not easily cleaned as the gel may adhere to them and leave residues [27].

In the broader sense of gel applications, the concept of blending two or more polymers inside the 3D complex of the final gel is largely known. The blending facilitates tailoring gels with specific properties in addition to producing gels with improved characteristics that can circumvent the possible limitations conveyed by gels made using unblended polymers [28-31]. In the conservation field, a number of gel blends have been developed. For instance, poly-2-hydroxyethyl methacrylate/polyvinyl pyrrolidone, polyvinyl alcohol/polyvinyl pyrrolidone, polyvinyl alcohol-borax blended with polyethylene oxide, and polyvinyl alcohol-borax complexed with carbomer. In all the above-mentioned hydrogel examples, the addition of the second polymer was done to optimize the properties of the resulting hydrogel to meet the criteria of specific cleaning needs [32-35].

In a previous study, we blended PVA-B with agarose and the resulting hydrogel proved to be efficient in removing deteriorated consolidants from ancient Egyptian wall paintings [36]. The PVA-B/AG double network (DN) hydrogel exhibited a good workability during application and was able to host different solvents such as acetone, 1-butanol, 1-pentanol, methyl ethyl ketone as cleaning agent. In this paper, we investigated in greater detail various characteristics of the PVA-B/AG double network hydrogel such as (a) its chemical structure, (b) 
liquid phase retention, (c) mechanical strength, (d) rheological properties, and e) self-healing behavior.

\section{Materials and methods}

\section{Materials}

Polyvinyl alcohol (PVA) (98.0-98.8\% hydrolyzed, M.W. 146,000-186,000) and Ethanol 96\% (EtOH) (technical) were purchased from Acros Organics. Di-Sodium tetraborate decahydrate (borax) (ACS, ISO reagent), Titriplex ${ }^{\circledR}$ III for analysis (EDTA) (ethylenediaminetetraacetic acid, disodium salt dihydrate), and Methylene blue were supplied by Merck. Agarose (AG) (molecular biology grade, low EEO/Multipurpose) was obtained from Fisher scientific. Acetone $\geq 99 \%$ (AC) (technical) was acquired from VWR chemicals. $p$-Xylene 99\% (Xyl) and RhodamineB 95\% (HPLC) were purchased from Sigma Aldrich. Ethomeen ${ }^{\circledR}$ C25 (C25) was obtained from Kremer pigment. All the chemicals were used as received.

\section{Hydrogel synthesis}

The hydrogels were prepared according to the procedure described in [36]. For more details, see Additional file 1: Hydrogel synthesis, Tables S1 and S2, and Fig. S2. Figure 1 depicts the stages of gel formation of the PVA-B/ AG double network hydrogel.

The measurements described below focused on hydrogel formulations consisting of 3\% PVA-B in water, $4 \%$ PVA-B in water, and $3 \% / 1 \%$ PVA-B/AG in water. This is to draw a comparison between increasing the concentration of PVA (from 3 to $4 \%$ ) in the PVA-B hydrogels. In addition to comparing between $4 \%$ PVA-B hydrogel and replacing $1 \%$ of PVA with AG (3\%/1\% PVA-B/AG). We also investigated the effects of adding increased levels of agarose to PVA-B systems on their rheological behavior. Moreover, PVA-B and PVA-B/AG hydrogels were loaded with different cleaning agents to study their influence on the characteristics of these two gel systems. Three organic solvents with different polarities were chosen for this reason: ethanol (with polarity index $P^{\prime}=5.1$, based on the scale proposed by Snyder [37]), acetone (5.4) and xylene (2.4). In addition, other cleaning agents such as the chelating agent EDTA and the surfactant Ethomeen $\mathrm{C} 25$ were studied.

\section{Chemical characterization}

Chemical characterization of PVA-B/AG was carried out using a Nicolet ${ }^{\mathrm{TM}}$ iS $^{\mathrm{TM}} 5$ Fourier transform infrared (FTIR) Spectrometer. ATR-FTIR spectra of pure PVA, 3\% PVA-B, 3\%/1\% PVA-B/AG, and 2\% AG were recorded. All hydrogel formulations were prepared and dried at room temperature to a constant weight to remove as much water as possible. The spectra collected were at a

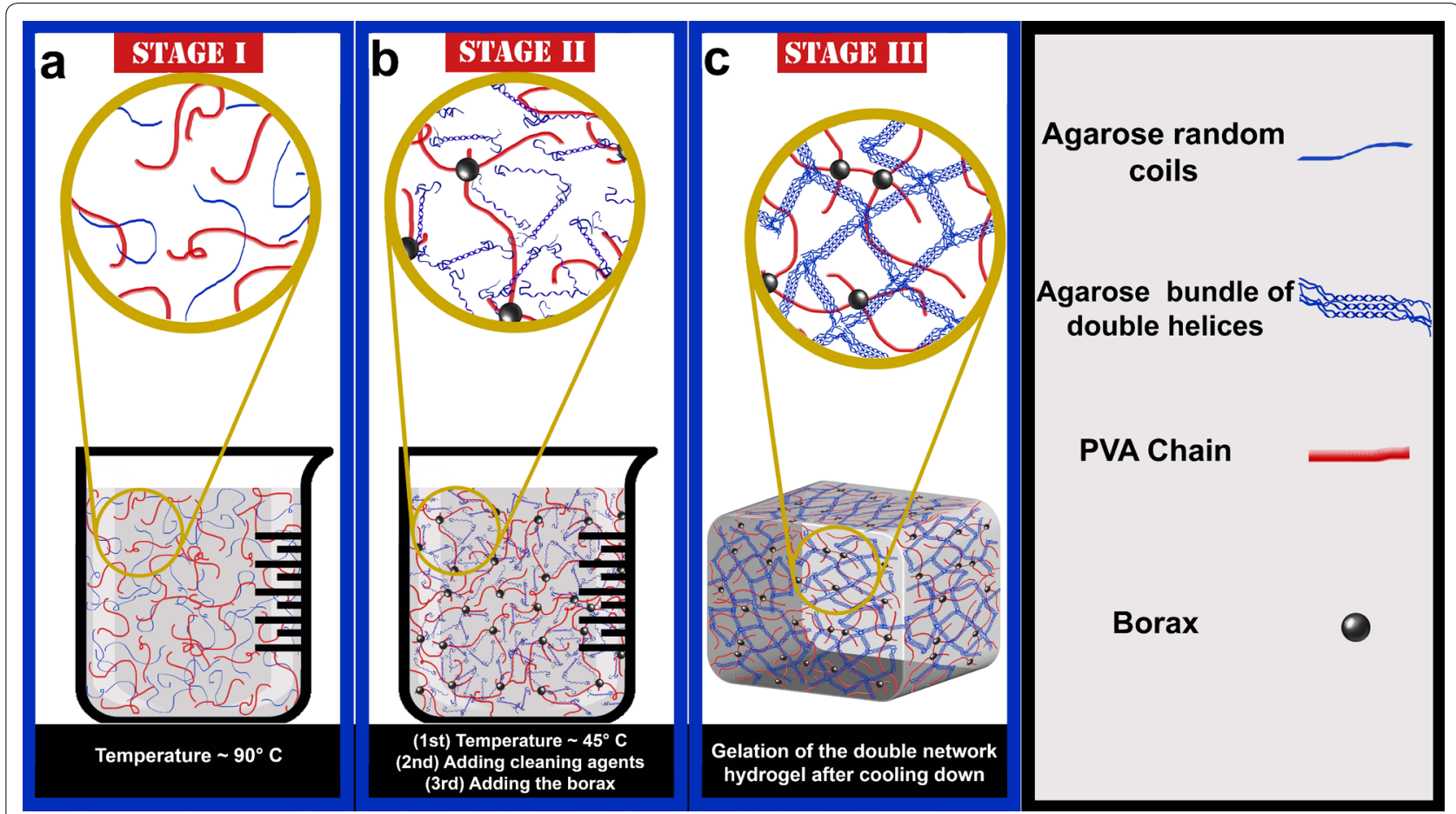

Fig. 1 Schematic illustration of preparation and gelation processes of PVA-B/AG DN hydrogel loaded with cleaning agents 
resolution of $4 \mathrm{~cm}^{-1}$ in the range of $4000-500 \mathrm{~cm}^{-1}$ using 32 scans. Each spectrum represents the average of five measurements.

\section{Liquid phase retention}

The hydrogel formulations were subjected to isothermal thermogravimetric measurements in order to investigate their ability to retain the volatile components. This was carried out on a thermogravimetric balance (TGA/DSC 3+, METTLER TOLEDO). A sample of approximately $98 \pm 13 \mathrm{mg}$ was placed in an aluminum cup $(100 \mu \mathrm{l})$ at $35{ }^{\circ} \mathrm{C}$ for $20 \mathrm{~min}$ under the flow of argon gas at $20 \mathrm{ml} /$ $\mathrm{min}$. The collected data are based on the average of three measurements for each hydrogel formulation. In this experiment, a comparison was made between 3\% PVAB, $4 \%$ PVA-B, and 3\%/1\% PVA-B/AG hydrogel formulations. In addition, the same formulations were loaded with $20 \% \mathrm{AC}, 20 \% \mathrm{EtOH}$ and $20 \% \mathrm{Xyl}$. The formulations that showed a lower mass loss are those that have a better ability to retain the liquids for a longer time and they permit better control of the volatile solvents [35].

\section{Liquid phase diffusion into porous surfaces}

In order to explore the penetration of the liquids released by the three hydrogel formulations (3\% PVA-B, $4 \%$ PVA$B$, and $3 \% / 1 \%$ PVA-B/AG), they were loaded with a Rhodamine $B$ dye $(0.1 \%)$. Slabs of hydrogel were placed on the surface of limestone tiles for $3 \mathrm{~h}$ and on plaster tiles for one hour. After that, the hydrogels were peeled off from the tiles' surface by means of tweezers. Cross sections were cut from the treated areas of the tiles using a hand saw. The tiles were cut carefully from the back side to avoid sawing into the treated areas and manipulating the dye which had penetrated into the tiles. The cross sections of the limestone tiles were examined under an Olympus SZX12 microscope equipped with a TOUP$\mathrm{CAM}^{\mathrm{TM}}$ (U3CMOS05100KPA) digital camera; the penetration depth of the dye was measured as the average value of 45 measurements on three different cross sections treated with the same hydrogel formulation. The cross sections of the plaster tiles were photographed using a Nikon D5600 camera and the depth of penetration was measured by processing the collected images in photoshop software.

\section{Morphological structure}

A FEI Quanta 250 FEG scanning electron microscope was used to study the microstructure and the morphology of the 3\% PVA-B, $4 \%$ PVA-B, and 3\%/1\% PVA-B/AG hydrogel formulations. Firstly, the hydrogels were frozen using dry ice for $2 \mathrm{~h}$ and then freeze-dried by means of an Alpha 1-2 LDplus instrument for eight days to remove as much water as possible. The hydrogel samples were sliced with a sharp blade and mounted on the SEM holder using conductive silver paint. A Leica EM ACE200 low vacuum coater was used to carbon-coat the samples.

\section{Mechanical strength}

Uniaxial tensile tests were carried out to study the mechanical properties of the hydrogels using a testing machine model LRXplus, Lloyds Instruments Ltd. The $4 \%$ PVA-B and 3\%/1\% PVA-B/AG hydrogel formulations were tested in order to explore the effect of the addition of agarose to PVA-B hydrogel compared to increasing the concentration of PVA-B from 3 to 4\%. Initially, the samples were fixed in a briquet mold for 5 min before being subjected to the measurement conditions. The two ends of the mold are in the shape of clips with an inner radius of $15.5 \mathrm{~mm}$ and the gauge length was $30 \mathrm{~mm}$ (see Additional file 1: Fig. S5). The machine was operated with a loading cell of $1000 \mathrm{~N}$ at a cross-head speed of $1000 \mathrm{~mm} /$ min. All the measurements were done at room temperature. The results are based on four measurements of each formulation.

\section{Rheological properties}

Rheological measurements were executed using an Anton-Paar Physica MCR 500 rheometer equipped with double Peltier temperature control unit. All tests were conducted using $25 \mathrm{~mm}$ diameter flat plates. The gap between the plates was kept constant at $1 \mathrm{~mm}$ for all the test. To guarantee the correct sample diameter, the following sample loading procedure was adopted: a piece of hydrogel, large enough to fill the gap between the plates, was placed on the lower plate. In a next step, the upper plate was lowered to a gap of $1.050 \mathrm{~mm}$. At this gap setting, the hydrogel sample was trimmed with a spatula so that the diameter of the sample was the same as the diameter of the plates. Afterwards, the gap was reduced with 50 microns to be exactly $1 \mathrm{~mm}$; assuring a slight outward bulge of the sample.

Frequency sweeps were recorded at $25{ }^{\circ} \mathrm{C}$, from 10 to $0.01 \mathrm{~Hz}$, and at a strain level of $4 \%$ (except for the formulation 3\%/1\% PVA-B/AG + 0.5\% EDTA, which were tested at a lower strain ' $1 \%$, to reduce the risk for slippage). The strain levels were selected from preliminary stress sweeps to be well within the linear viscoelastic range. The measurements were performed on the following hydrogel formulations: 3\% PVA-B, 4\% PVAB, 3\%/0.5\% PVA-B/AG, 3\%/1\% PVA-B/AG, 3\%/1.5\% PVA-B/AG, $4 \% / 1 \%$ PVA-B/AG, and $2 \% / 1.5 \%$ PVA-B/ AG. In addition, $3 \% / 1 \%$ PVA-B/AG hydrogel was loaded with $20 \% \mathrm{AC}, 20 \% \mathrm{EtOH}, 20 \% \mathrm{Xyl}, 0.5 \%$ EDTA, and $5 \% \mathrm{C} 25$. This was to study the influence of these additives on PVA-B/AG DN. The collected curves represent the average of at least three repeats. It is worth 
mentioning that the hydrogel formulations loaded with xylene and EDTA (suffering from syneresis) were rapidly blotted by tissue paper to remove any excess of liquids on the sample's surface prior to measuring. This was done to avoid sample slippage during the measurement. It should be noted that the blotting may modify the measured $G^{\prime}$ and $G^{\prime \prime}$ values to some extent. The standard deviation is calculated for all the investigated samples and presented in the frequency sweeps and creep curves as error bars.

Creep tests were performed by applying a constant shear stress of $15 \mathrm{~Pa}$ on the sample at $25^{\circ} \mathrm{C}$. The variation of the strain in response to the applied force was measured over approximately $6100 \mathrm{~s}$. Two repeats were collected for each hydrogel formulation.

\section{Vertical flow test}

This test was designed to probe the shape stability of hydrogels when they are applied in a vertical position. The hydrogel formulations 3\% PVA-B, 4\% PVA-B, and $3 \% / 1 \%$ PVA-B/AG were investigated with only water in addition to others loaded with $20 \% \mathrm{AC}, 20 \% \mathrm{EtOH}$, and $20 \%$ Xyl. The hydrogel formulations were dyed with methylene blue $(0.001 \%)$ for better visibility and all cut using a shape cutter in a specific shape with slab thickness of $c .3 \mathrm{~mm}$. Subsequently, they were applied on a graded sheet fixed on a wall so that the hydrogel slabs were on the same height level. The hydrogel formulations were photographed at the following time intervals: 0,10 , 30, 60 and $360 \mathrm{~min}$. Temperature and relative humidity, during the test, were $22 \pm 1{ }^{\circ} \mathrm{C}$ and $62 \pm 2 \%$ respectively.

\section{Self-healing behavior}

To investigate the self-healing behavior macroscopically, the hydrogels (3\% PVA-B, 4\% PVA-B, and 3\%/1\% PVA-B/ $A G)$ were dyed with either rhodamine $B$ or methylene blue to obtain a better optical contrast between two adjacent pieces. The hydrogels were cut into pieces, using a blade. Differently colored hydrogel pieces were placed together into contact for $10 \mathrm{~min}$ to allow the healing process to take place at room temperature. To verify the attachment of the healed pieces, the hydrogels were vertically suspended by means of tweezers.

In addition, to determine the speed of the healing behavior of each hydrogel, they were examined under the microscope. In this test, 3\% PVA-B and 3\%/1\% PVA-B/ AG hydrogel formulations were dyed with methylene blue for better visibility and cast in the form of disks. Under the microscope, a cut in the hydrogel was made using a sharp blade and a video was recorded to track the speed of the healing process of the cut over time.

\section{Results and discussion}

\section{Chemical characterization}

In all the investigated samples, there is a small band at $\sim 1640 \mathrm{~cm}^{-1}$ with a small intensity which may be attributed to $\mathrm{O}-\mathrm{H}$ bending due to the absorption of water. Water also has a broad band at $\sim 3400 \mathrm{~cm}^{-1}$ [3], so it is expected that the band at $\sim 3270 \mathrm{~cm}^{-1}$ might also have a contribution from water. However, since the peak at $\sim 1640 \mathrm{~cm}^{-1}$ has a constant intensity in the samples, we assume that the changes observable in the band at $\sim 3270 \mathrm{~cm}^{-1}$, which is present in the collected spectra, are not linked to the contribution of water (see Fig. 2a). In the PVA spectrum, the asymmetrical and symmetrical $\mathrm{CH}_{2}$ stretching vibrations appear at 2938 and $2910 \mathrm{~cm}^{-1}$, respectively. The same spectrum shows the peaks at 1708 and $1560 \mathrm{~cm}^{-1}$ which represent stretching carbonyl group $(\mathrm{C}=\mathrm{O})$ of the acetate group. This originates from remains of the manufacturing of PVA through hydrolysis of polyvinyl acetate. The $\mathrm{C}-\mathrm{H}$ bending peak of $\mathrm{CH}_{2}$ group appears at $1414 \mathrm{~cm}^{-1}$. The peaks at 1376 and $1239 \mathrm{~cm}^{-1}$ correspond to the rocking of methyl groups $\left(-\mathrm{CH}_{3}\right)$ or $-\mathrm{CH}_{2}$ wagging vibrations, respectively while the peak at $1327 \mathrm{~cm}^{-1}$ is attributed to $\mathrm{H}-\mathrm{C}-\mathrm{H}$ and $\mathrm{C}-\mathrm{O}-\mathrm{H}$ bending vibrations. The significant peak at $1141 \mathrm{~cm}^{-1}$, corresponding to $\mathrm{C}-\mathrm{C}$ and $\mathrm{C}-\mathrm{O}-\mathrm{C}$ stretching vibrations, is indicative of the crystallinity of PVA. The $\mathrm{C}-\mathrm{O}$ stretching vibration occurs at $1085 \mathrm{~cm}^{-1}$ and the peak at $916 \mathrm{~cm}^{-1}$ is attributed to the $-\mathrm{CH}_{2}$ stretching vibration. The vibration at $832 \mathrm{~cm}^{-1}$ is assigned to $\mathrm{C}-\mathrm{C}$ stretching and $\mathrm{C}-\mathrm{H}$ out-of-plane vibrations [38-40].

As reported, a chemical interaction between two materials after mixing them can lead to changes in the resulting ATR-FTIR spectrum. These changes may consist of shifts in the characteristic peaks or the appearance of new ones [41]. In our case, when borax is added to PVA solution, the resulting film shows significant changes to the $\mathrm{O}-\mathrm{H}$ broad band of pure PVA; the band becomes broader with a lower intensity and shifts to higher wavenumbers which is associated with the interaction between PVA and borax (see Fig. 2a) [42]. In addition, there is a slight shift occurring in the characteristic peaks of pure PVA. The ATR-FTIR spectrum of PVA-B also confirms the presence of the asymmetric stretching relaxation of B-O-C at 1420 and $1334 \mathrm{~cm}^{-1}$, indicating the crosslinking of PVA with borate ions through the formation of tetrahedral complexes. The peaks at 830 and $661 \mathrm{~cm}^{-1}$ are attributed to $\mathrm{B}-\mathrm{O}$ stretching from the residual of $\mathrm{B}(\mathrm{OH})_{4}{ }^{-}$and bending of $\mathrm{B}-\mathrm{O}-\mathrm{B}$ linkages within borate networks, respectively [43].

The ATR-FTIR spectrum of PVA-B/AG blend shows the characteristic bands of agarose such as the $\mathrm{C}-\mathrm{O}-\mathrm{C}$ and glycosidic linkage at $1069 \mathrm{~cm}^{-1}$. The absorption bands at 931 and $893 \mathrm{~cm}^{-1}$ are associated with 


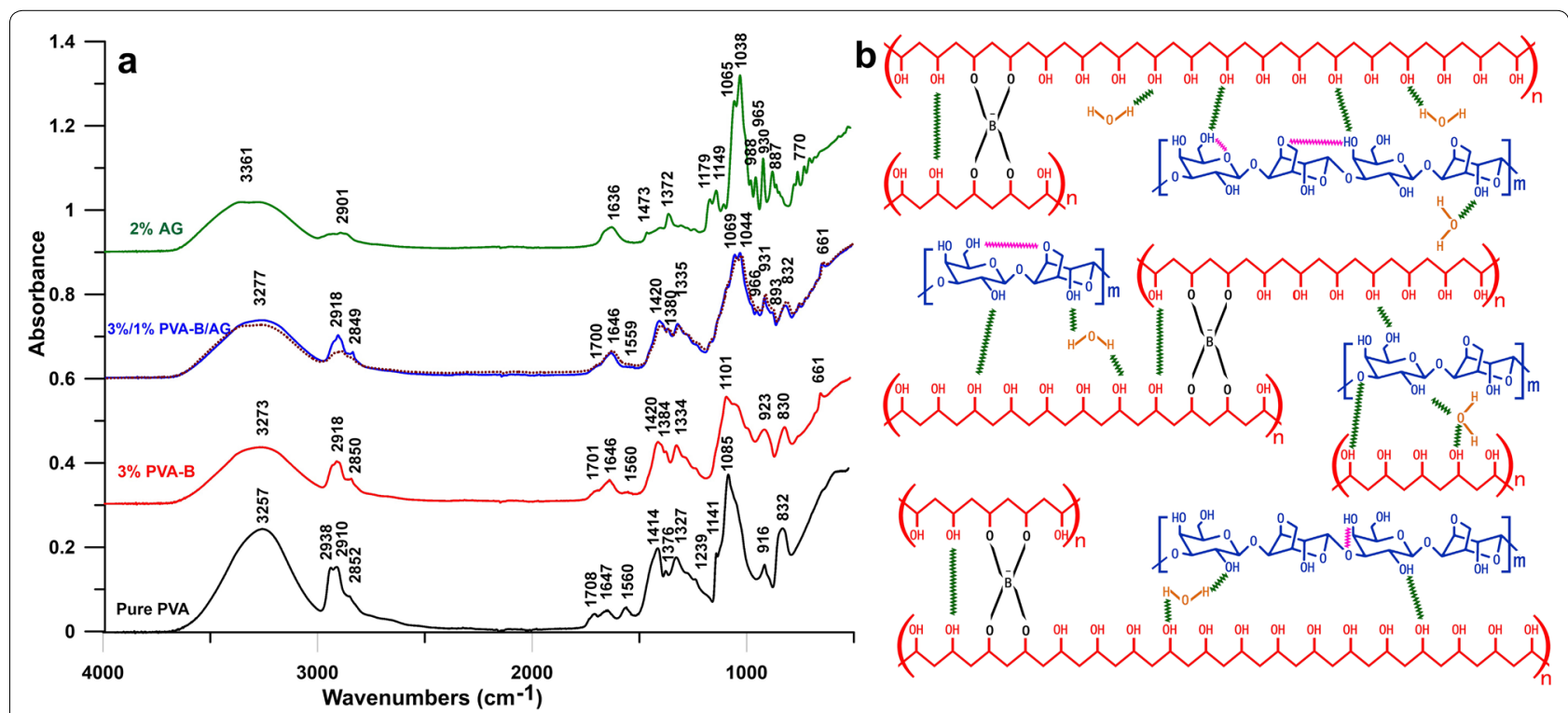

Fig. 2 a FTIR spectra of pure PVA, 3\% PVA-B, 3\%/1\% PVA-B/AG, and 2\% AG. The brown dotted spectrum represents the sum of 3\% PVA-B and 2\% AG spectra. $\mathbf{b}$ Illustration of the proposed interactions between PVA (in red), borax (in black), AG (in blue), and water (in orange) in the PVA-B/AG DN hydrogel and the formation of intermolecular (in green) and intramolecular (in magenta) hydrogen bonds

3,6-anhydrogalactose and the $\mathrm{C}-\mathrm{H}$ bending vibrations of anomeric carbon in $\beta$-galactose residues, respectively [44-46]. Upon mixing PVA-B with AG, the hydroxyl broad band changes in intensity and position. In addition, other slight changes in the intensity and peak position of characteristic PVA-B and AG peaks are observed. For instance, the two peaks of AG at 1065 and $1038 \mathrm{~cm}^{-1}$ shift to higher frequencies at 1069 and $1044 \mathrm{~cm}^{-1}$; these changes reflect the formation of intermolecular hydrogen bonds between PVA-B and AG (see Fig. 2b) $[47,48]$.

\section{Liquid phase retention}

Figure 3 summarizes the mass loss of different hydrogel formulations after $20 \mathrm{~min}$ in the thermogravimetric balance. When no solvents other than water are present, $3 \%$ PVA-B loses liquid faster than $4 \%$ PVA-B and 3\%/1\% PVA-B/AG. On the other hand, $4 \%$ PVA-B has a slight advantage in retaining liquid over $3 \% / 1 \%$ PVA-B/AG. These results can be confirmed by the those obtained from the SEM micrographs of the same hydrogel formulations (see "Morphological structure" section). The samples with smaller size of the pores the better they would retain the liquids. For example, the $4 \%$ PVA-B hydrogel retained the liquids better than the 3\% PVA-B and 3\%/1\% PVA-B/AG hydrogels because its small pores' size. The same behavior is observed for the hydrogel formulations loaded with solvents. However, due to the tendency of xylene of becoming expelled, the corresponding formulations does not follow the same

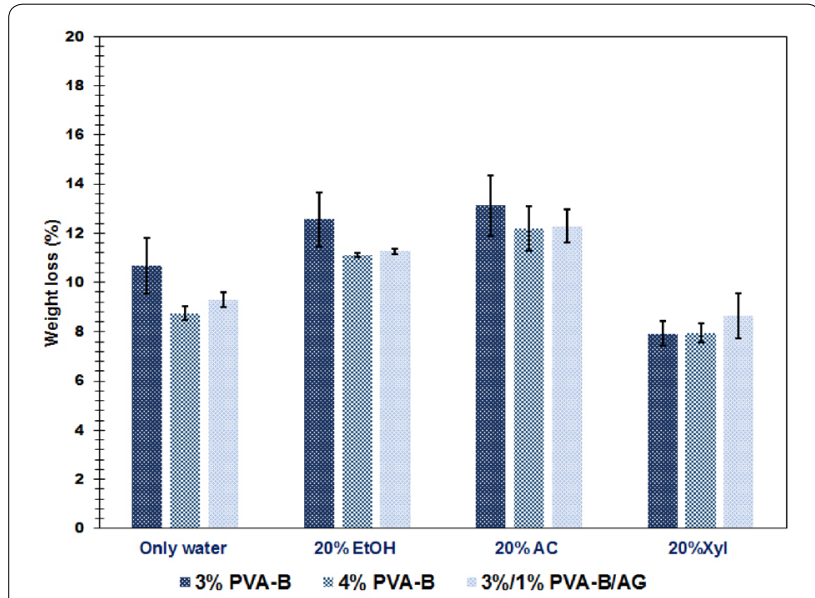

Fig. 3 Liquid phase retention of 3\% PVA-B, 4\% PVA-B, and 3\%/1\% PVA-B/AG hydrogel formulations with and without solvents. The error bars represent the standard deviation

trend. The retained concentrations of $20 \%$ xylene by the $3 \%$ PVA-B, $4 \%$ PVA-B, and 3\%/1\% PVA-B/AG hydrogel formulations are as follows: $13.5 \%, 17.5 \%$, and $19 \%$ respectively. The weight loss of all the hydrogel formulations follows an inverse trend relative to the boiling points of the three investigated organic solvents (water: $100{ }^{\circ} \mathrm{C}$, EtOH: $78{ }^{\circ} \mathrm{C}, \mathrm{AC}: 56{ }^{\circ} \mathrm{C}$, and Xyl: $139-142{ }^{\circ} \mathrm{C}$ ) [49]. 


\section{Liquid phase diffusion into porous surfaces}

The results obtained by means of this test are similar to those of the liquid phase retention test (see Additional file 1: Fig. S3 and Additional file 1: Fig. S4). The formulations characterized by a low liquid retention cause more rhodamine $B$ dye to diffuse than those with a high liquid retention. In this case, 3\% PVA-B shows the most important diffusion of the dye; then $3 \% / 1 \%$ PVA-B/AG and $4 \%$ PVA-B. There is a minor difference between the hydrogel formulations 4\% PVA-B and 3\%/1\% PVA-B/AG. As expected, the diffusion into the plaster tiles is considerably higher than that into the limestone ones due to the high porosity of the plaster tiles, even at different application times.

It is worth noting that during peeling off of the hydrogels from the surface of the plaster tiles, both PVA-B formulations (3\% and $4 \%)$ strongly adhered to the tiles and left behind a layer of the hydrogel (see Fig. 4). Only the $3 \% / 1 \%$ PVA-B/AG DN hydrogel did not show that

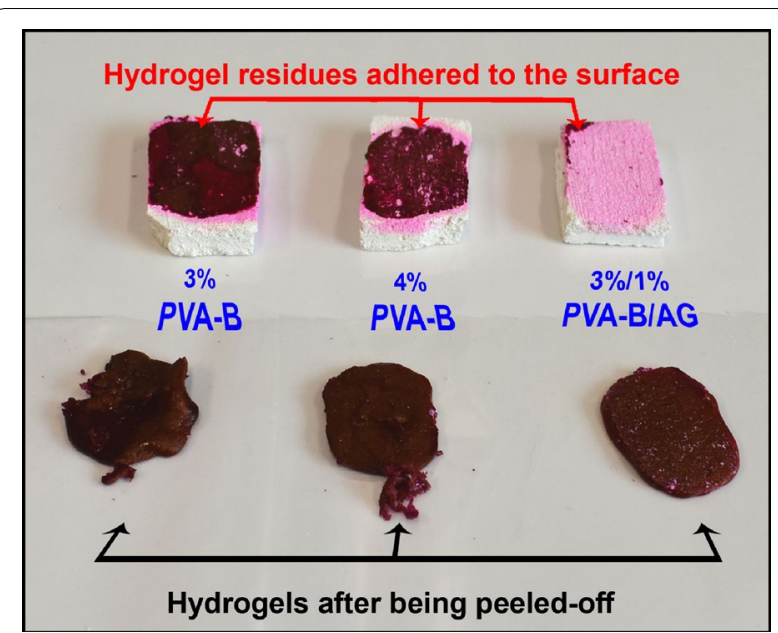

Fig. 4 Breakage and residues left on the surface by the 3\% PVA-B and 4\% PVA-B formulations after an hour application on plaster tiles while the 3\%/1\% PVA-B/AG hydrogel remained intact and did not adhere to the treated surface problem; the hydrogel slab could be peeled off almost intact. The process was repeated with the formulations loaded with the three solvents (AC, EtOH, and $\mathrm{Xyl}$ ) and again the hydrogels without the agarose adhered to the plaster tiles while the agarose-containing ones did not. This can be related to difference in the mechanical properties of the PVA-B formulations and the PVA-B/AG double network. In order to test the possible differences in mechanical properties between pure PVA-B and the PVA-B/AG double network, it was decided to perform tensile tests.

\section{Morphological structure}

Figure 5a illustrates the large interconnected pores of the 3D network of 3\% PVA-B hydrogel. When agarose is added to the formulation, the thickness of the pore walls is strengthened, leading to a reduction in the size of the pores (see Fig. 5b). In contrast, the 4\% PVA-B hydrogel shows a large number of pores with a smaller size than that of 3\% PVA-B and 3\%/1\% PVA-B/AG hydrogel (see Fig. 5c).

\section{Mechanical strength}

The four replicates of 4\% PVA-B all broke at different elongation points whereas the $3 \% / 1 \%$ PVA-B/AG hydrogel did not break in any of the four measurements, allowing the machine to reach its maximum gauge length (see Fig. 6, Additional file 1: Fig. S5, Additional files 2 and 3 ). These results indicate that the 4\% PVA-B hydrogel is less flexible at these large elongations than the 3\%/1\% PVA-B/AG DN hydrogel. Recently, in combination with other materials, this DN gel was used by Kim et al. [30] to create a high-performance self-healing flexible planar supercapacitor. The superior mechanical strength exhibited by the PVA-B/AG DN hydrogel may be ascribed to: (a) the hydrogen bonds formed between the PVA-B system and the AG polymers inside this hydrogel [30], (b) the strengthened walls of the pores of the DN hydrogel. These results confirm the difference in behavior noticed

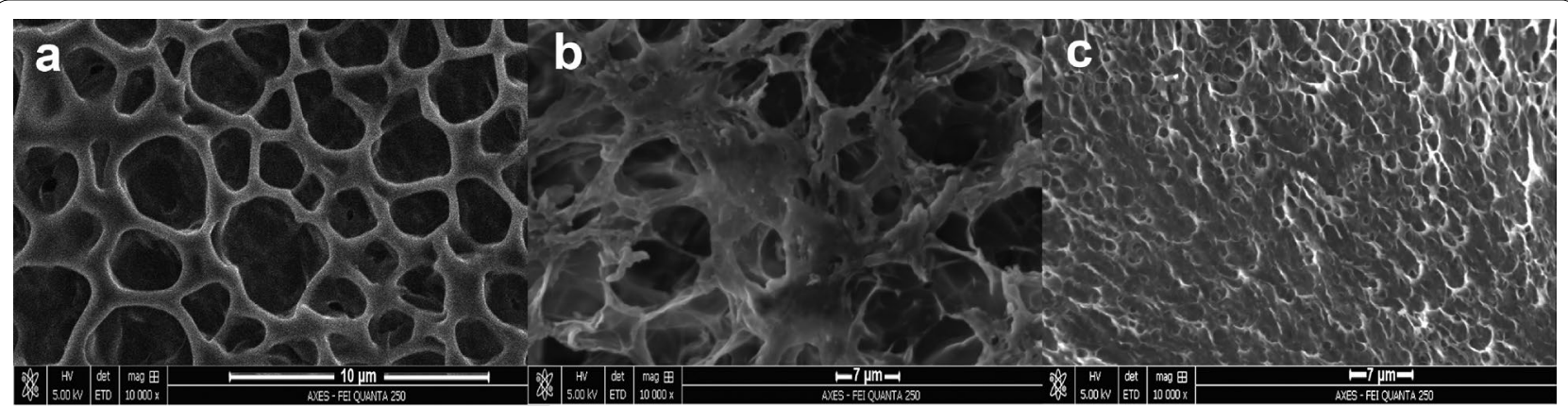

Fig. 5 SEM micrographs $(10,000 \times)$ showing the microstructure of the hydrogels. a 3\% PVA-B, b 3\%/1\% PVA-B/AG DN, and c 4\% PVA-B 

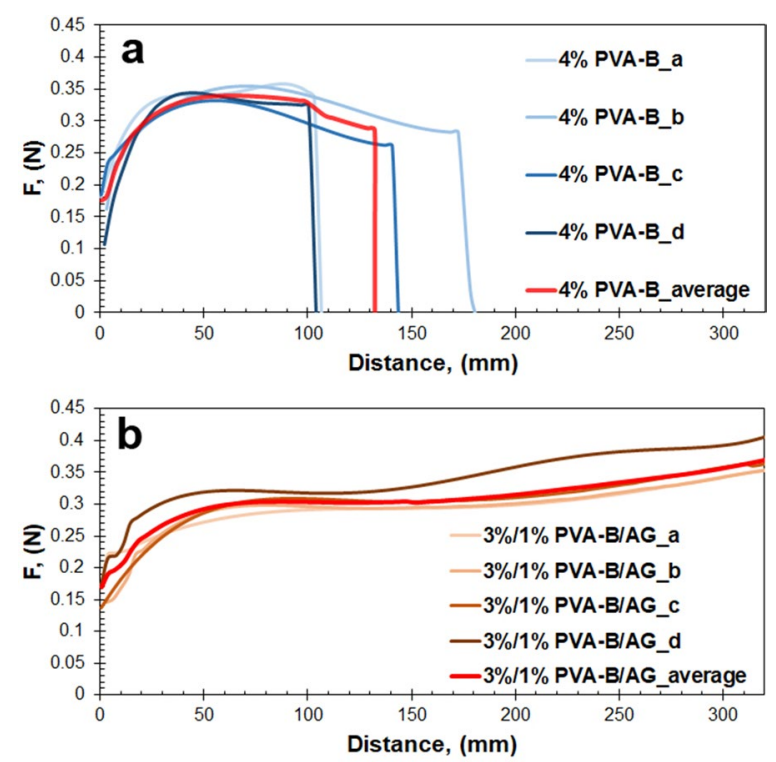

Fig. 6 The elongation of 4\% PVA-B and 3\%/1\% PVA-B/AG hydrogels. The data are based on four measurements

in "Liquid phase diffusion into porous surfaces" section where the 3\% PVA-B and 4\% PVA-B hydrogels were damaged during peeling off from the plaster tiles while the $3 \% / 1 \%$ PVA-B/AG DN hydrogel could be completely peeled off in one piece.

\section{Rheological properties}

Oscillatory shear measurements were carried out to study the effect of AG on PVA-B systems when they are blended together. The elastic storage modulus ( $\mathrm{G}^{\prime}$, a measure of the elastic response of a material) and loss modulus ( $G^{\prime \prime}$, a measure of the viscous response of a material) were measured. When $G^{\prime}$ is larger than $G^{\prime \prime}$, the material exhibits elastic behavior while when $G^{\prime}<G^{\prime \prime}$, the material shows viscous behavior [26]. The frequency sweep curves for all measured hydrogel formulations are plotted in Additional file 1: Figs. S6 and S7. From the frequency sweep curves, the intrinsic elastic modulus $\left(G_{0}^{\prime}\right)$ and apparent relaxation time $\left(\tau_{c}\right)$ can be obtained. $G_{0}^{\prime}$ is calculated based on the average of the last three highest frequency points of $G^{\prime} . \tau_{c}$ is obtained from the crossover frequency point $\left(\omega_{c}\right)$ of the two moduli $\left(G^{\prime}\right.$ and $\left.G^{\prime \prime}\right)$, according to: $\left(\tau_{c}=2 \pi / \omega_{c}\right) . G_{0}^{\prime}$ is referred to as the 'stiffness' of the system and it should be higher than $400 \mathrm{~Pa}$ to provide enough elasticity of the hydrogel to be peeled off [35]. On the other hand, when the crossover point shifts to lower frequencies, $\tau_{c}$ increases and the hydrogel shows a better shape stability [50] (see Additional file 1: Fig. S8).
PVA-B systems are viscoelastic; at high frequencies (or short loading times) the behavior is elastic and the storage modulus (G) is dominating the behavior. At lower frequencies (or longer loading times), the loss modulus $\left(G^{\prime \prime}\right)$ or the viscous behavior is dominating. In accordance with the literature [26], upon increasing the concentration of PVA, the hydrogels become stiffer at high frequencies and show a higher viscosity in the low frequency domain (see Fig. 7a).

In Fig. 7b and Additional file 1: Fig. S8a, data recorded for the hydrogel formulations containing a constant amount of PVA-B and increasing amounts of AG, are plotted. The data show that increasing AG also leads to a stiffer behavior, a higher storage and loss modulus at all frequencies investigated. The same trend can be observed on the $\tau_{c}$ data (see Additional file 1: Fig. S8b). In Fig. 7c, d hydrogel formulations with various amounts of both components are plotted; in Fig. 7c, the comparison between using $4 \%$ of PVA-B versus replacing $1 \%$ with AG is obvious. Both hydrogel formulations (4\% PVA-B and $3 \% / 1 \%$ PVA-B/AG) behave similar in this test. However, the 3\%/1\% PVA-B/AG hydrogel formulation has a slightly higher $\tau_{c}$ value than the 4\% PVA-B hydrogel (see Additional file 1: Fig. S8b). In Fig. 7d, a similar comparison is shown, again the effect of $1 \%$ change in the concentrations of PVA-B and AG is studied, this time at a lower concentration level; 3\%/0.5\% PVA-B/AG is compared to $2 \% / 1.5 \%$ PVA-B/AG. In this case, it is clear that these samples are not similar. The high frequency behavior is again rather similar, but from around $0.2 \mathrm{~Hz}$ and lower differences are obvious. The hydrogel formulation 3\%/0.5\% PVA-B/AG behaves as expected, there is a crossover of storage and loss moduli, indicating that the sample is moving into a flow region. This is not the case for sample $2 \% / 1.5 \%$ PVA-B/AG. In this sample, the storage modulus curve remains above the loss modulus curve; therefore, there is no crossover. As the frequency reduces, the storage modulus moves into a stable region. This is typical for the existence of crosslinks reducing further flow. Although the 2\%/1.5\% PVA-B/AG hydrogel formulation resembles the behavior of a true gel, when considering the measured $\mathrm{G}_{0}^{\prime}$ values, it is a flexible hydrogel.

Upon adding cleaning agents to the $3 \% / 1 \%$ PVA-B/ AG DN, the frequency sweep curves exhibit differences in $\mathrm{G}_{0}^{\prime}$ and $\tau_{c}$ data (see Additional file 1: Figs. S7 and S9). All the cleaning agents added to the formulation induced increases in $G_{0}^{\prime}$ and $\tau_{c}$ values except the formulation loaded with $5 \% \mathrm{C} 25$. This formulation only shows minor differences in response compared to $3 \% / 1 \%$ PVA-B/AG with no cleaning agents included. The aforementioned increase agrees with the results presented in [24, 35, 51]. The inclusion of organic liquids in PVA-B systems 

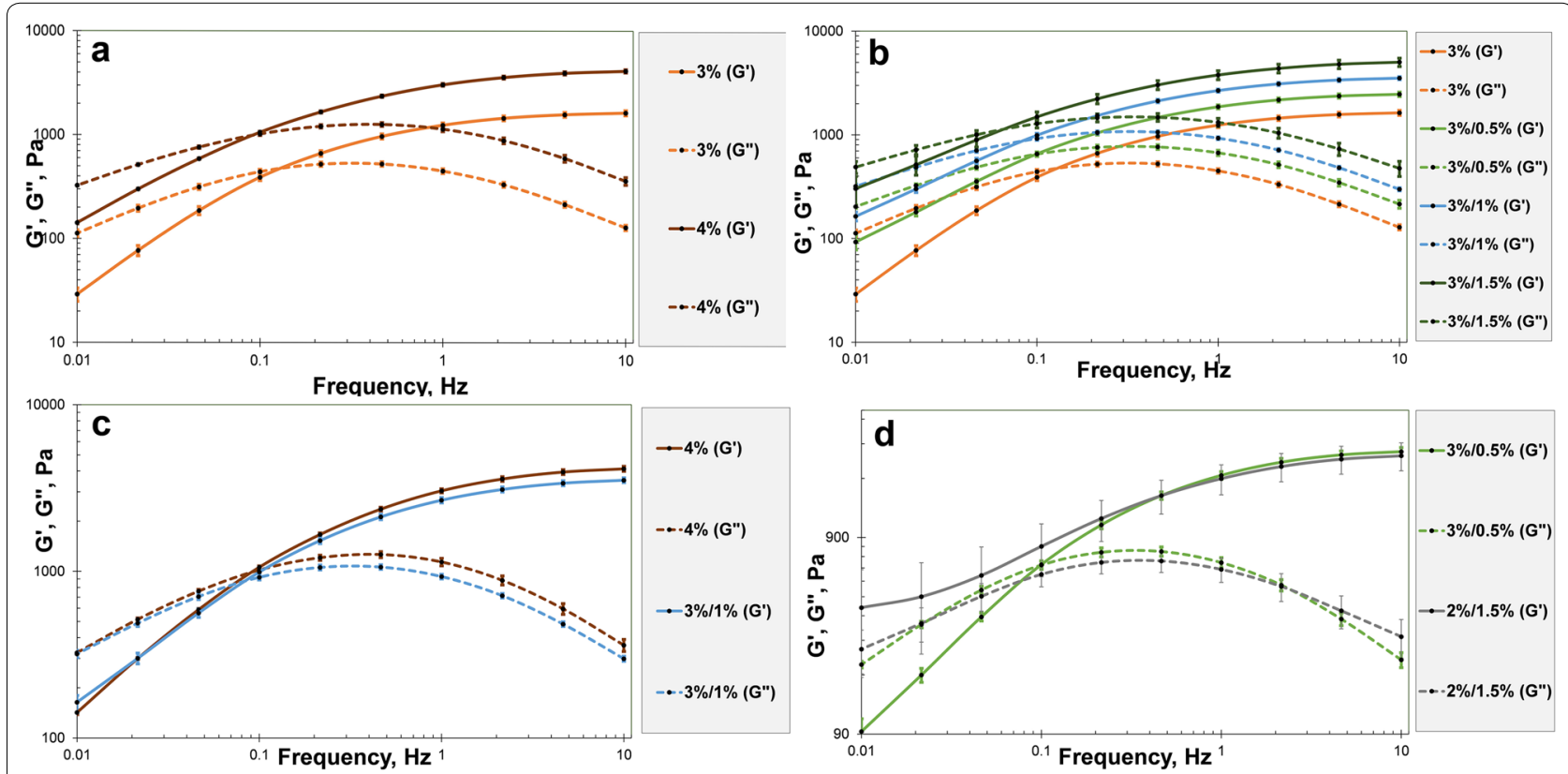

Fig. 7 Frequency sweeps at $25^{\circ} \mathrm{C}$. a Hydrogel formulations containing only PVA-B ( $3 \%$ and $4 \%$ ), b hydrogel formulations containing $3 \%$ PVA-B and increasing concentration of $A G, \mathbf{c}, \mathbf{d}$ Hydrogel formulations containing various percentages of PVA-B and $A G$. The standard deviations are presented as error bars

strengthens the hydrogel network, as the organic solvents stimulate the boron ions to bind to the PVA chains. The noticeable increase in the intrinsic storage modulus when EDTA is loaded into the hydrogel may be ascribed to the formation of hydrogen bonds between some of the EDTA ions and PVA chains in the PVA-B system [26]. The $\mathrm{G}_{0}^{\prime}$ measurements seem to suggest that the loading of PVA-B gels with increasingly low polarity solvents, produces stiffer hydrogels which can be due to the syneresis effect. More investigations are needed to confirm this observation. There is no clear correlation between $\tau_{c}$ and the polarity of the loaded solvents. Moreover, the $G^{\prime}$ curve of formulation, loaded with $0.5 \%$ EDTA, is higher than $G^{\prime \prime}$ all over the frequency range, indicating more gel-like behavior.

It was decided to perform creep tests to measure the behavior at long timescales, as opposed to frequency sweeps which are very suited to measure at short time scales. Some results are plotted in Fig. 8a-d, where the strain is plotted versus the creep time. In Fig. 8a, increasing the concentration of the PVA-B increases the stiffness or reduces the deformation seen in the creep test. It is also observed that the slope of the creep curve becomes constant at longer timescales, which is typical for viscoelastic materials [52]. If the strain versus creep time reaches a constant slope, this indicates that a steady state condition has been reached and the inverse of this slope corresponds to a zero-shear viscosity level. In Fig. 8b, creep curves are shown for samples containing a fixed amount of PVA-B and increasing concentrations of AG. In this case the effect of adding AG is very pronounced and it changes the shape of the creep curves. After adding AG, the creep curves resemble those of viscoelastic solids. The slope levels off with time, indicating that flow has almost stopped. 3\%/0.5\% PVA-B/AG hydrogel formulation shows this behavior and adding more AG strengthens this effect. This can be attributed to the additional network formed by AG and the strengthened walls of the pores.

In Fig. 8c, d, various concentrations of both components (PVA-B and AG) are compared; $(4 \%, 3 \% / 1 \%$, and $4 \% / 1 \%$, ) and $(3 \% / 0.5 \%, 3 \% / 1.5 \%$, and $2 \% / 1.5 \%)$ respectively. For example, when comparing the 4\% PVA-B sample to the $3 \% / 1 \%$ PVA-B/AG sample, the creep data clearly show that replacing $1 \%$ of PVA-B with AG is not equivalent; the creep curves clearly differ. A similar effect can be observed when comparing the $3 \% / 0.5 \%$ to the $2 \% / 1.5 \%$ PVA-B/AG hydrogel formulations. In addition, it is interesting to note that decreasing the concentration of PVA-B while using a fixed concentration of AG, appears to have a stronger effect. For example, when comparing $3 \% / 1 \%$ PVA-B/AG to $4 \% / 1 \%$ PVA-B/AG, the $3 \% / 1 \%$ PVA-B/AG hydrogel reaches a lower end strain; so it is stiffer as compared to $4 \% / 1 \%$ PVA-B/AG hydrogel (see Fig. 8c). And similarly, when comparing 3\%/1.5\% PVA-B/ AG and 2\%/1.5\% PVA-B/AG hydrogel formulations, the 

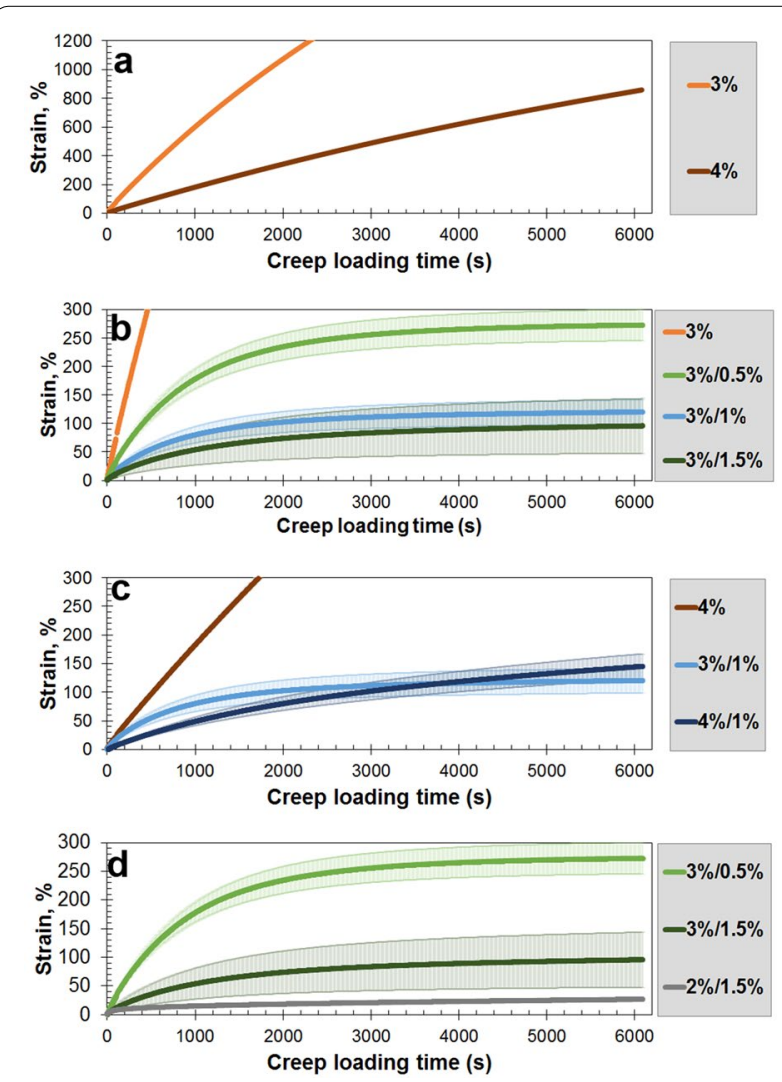

Fig. 8 Creep test results at $25^{\circ} \mathrm{C}$ : a Hydrogel formulations containing only PVA-B ( $3 \%$ and $4 \%)$, b hydrogel formulations containing $3 \%$ PVA-B and increasing concentrations of $A G, \mathbf{c}, \mathbf{d}$ Hydrogel formulations containing various percentages of PVA-B and AG. The standard deviations are presented as error bars
2\%/1.5\% PVA-B/AG hydrogel has undergone less deformation as the 3\%/1.5\% PVA-B/AG hydrogel (see Fig. 8d). This could indicate that the crosslinks formed by AG are more effective or more evenly distributed if the concentration of PVA-B is lower. We note that the reproducibility for all oscillation and creep tests decreases when adding more AG to the hydrogel formulations.

Finally, an empirical relation between creep and frequency sweeps was established, expressing the time range of the creep test as a frequency range. The strain can be transformed into a modulus using the constant stress/ creep strain. In Fig. 9, where the creep data is overlaid on the frequency sweep data, it can be seen that the empirical relation is valid for these materials. When comparing samples with the same total concentration of gellant (i.e. the sum of PVA-B and AG), it is clear that the samples show the same behavior at high frequency but deviate at low frequencies. In practice, when hydrogels of this type are applied on the surface for typically 10 to $30 \mathrm{~min}$ in the frequency range from 0.00027 to $0.00050 \mathrm{~Hz}$, differences in the rheological behavior of the hydrogels are observed.

\section{Vertical flow test}

The stability of a hydrogel in the vertical position is an important aspect especially when it is applied on a wall for long time. Flowing of the hydrogel negates the feature of selective application, in this case, the hydrogel reaches undesired areas of the surface to be treated. In this test, the $3 \% / 1 \%$ PVA-B/AG hydrogel shows a better shape stability in the vertical position than the 4\% PVA-B hydrogel (see Additional file 1: Fig. S10). The 3\% PVA-B formulation shows the least shape stability and flows significantly.

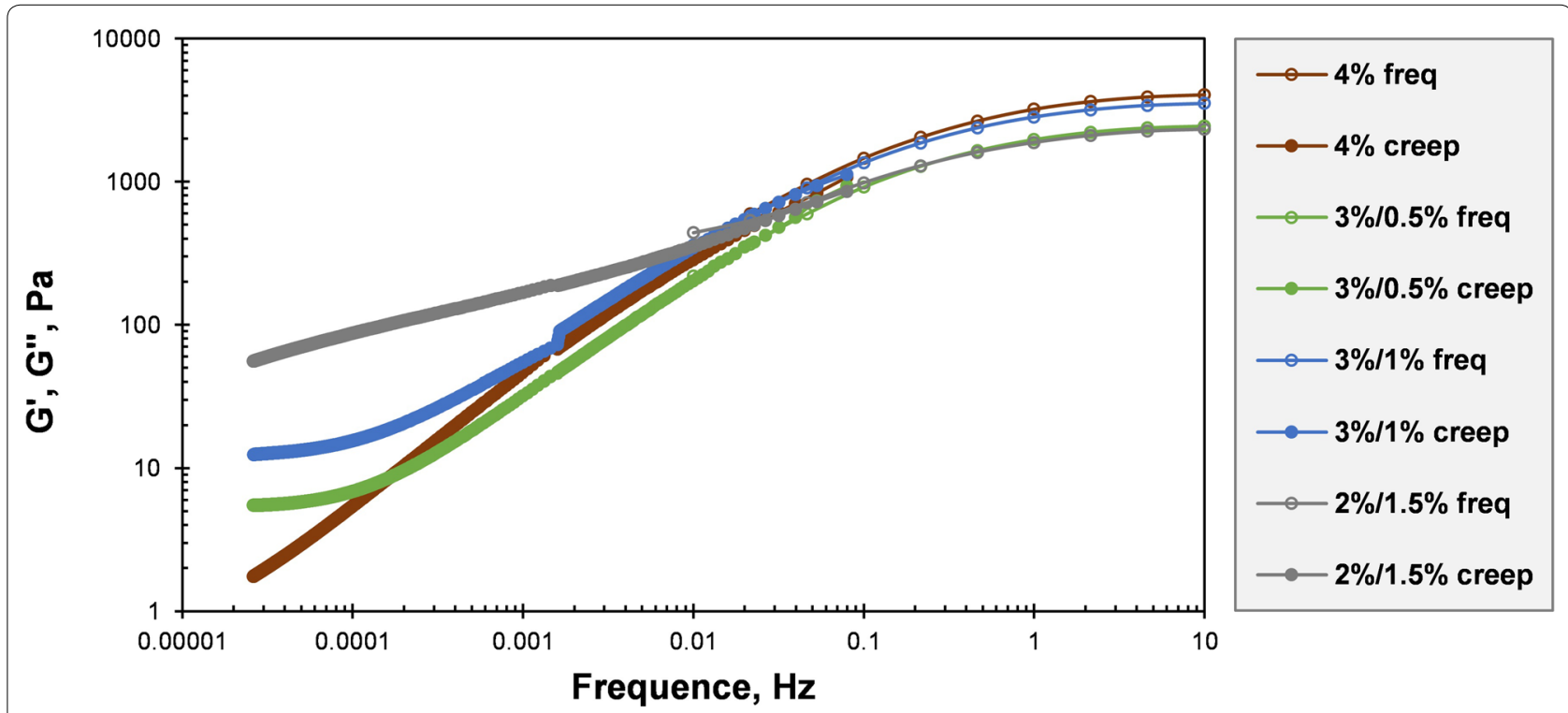

Fig. 9 Combined modulus frequency data and creep tests. Selected hydrogel formulations containing various concentrations of PVA-B and AG 
As a general observation, the addition of solvents hinders the flow behavior and this coincides with the results collected from oscillation and creep measurements. However, the results of the formulations loaded with Xyl exhibit different results than those collected from the frequency sweep tests. In the flow test, the hydrogels loaded with Xyl have better shape stability than those loaded with $\mathrm{AC}$ and $\mathrm{EtOH}$ which is not the case in the data of $\tau_{c}$ (see Additional file 1: Fig. S9). These differences can be ascribed to the expelled Xyl during the preparation of the hydrogels (see Additional file 1: Table S3). It should be taken into account that the degree of flow is also dependent on the size and/or the thickness of the hydrogel slab to be applied.

\section{Self-healing behavior}

This self-healing character can be beneficial in some cleaning treatments such as the cleaning of $3 \mathrm{D}$ objects. It is possible to cut the hydrogel into pieces and assemble them on the surface of the object to allow a good contact to the whole surface including all the curves. In a few minutes, the pieces will heal and form one single piece to cover the entire area to be treated. Later, the hydrogel can be cut and peeled off piece by piece. The exposed area can be cleared with a cotton swab to remove swollen/softened deposits. Moreover, if the hydrogel pieces are still usable after the first application, they can be put together to heal as a big single piece again that can be recut for another second application.

PVA-B hydrogels have certain self-healing characteristics due to the dynamic nature of the didiol complexation in the network. The labile bonds formed between tetrahedral borate ions and $\mathrm{OH}$-groups of PVA chains can be easily broken and reformed under ambient conditions (see Fig. 10a). Furthermore, the mobility of PVA strands and free borate ions facilitates the bonds formation across the contact interface and, consequently, the self-healing process takes place [53-55].

Based on the macroscopic tests, it is obvious that the PVA-B/AG hydrogel has similar healing properties as PVA-B hydrogels. For both 3\% PVA-B and 3\%/1\% PVA-B/ AG hydrogels, when the dyed hydrogel pieces were brought together, they healed into one piece under ambient conditions within ten minutes and without external stimuli. This was confirmed by lifting the hydrogels vertically (see Fig. 10b, c).

Microscopically, the speed of the self-healing character in the PVA-B/AG DN hydrogel (c. $4 \mathrm{~min}$ ) is slower than that of PVA-B hydrogel (c. 2 min), which may be ascribed to the fact that the agarose hinders the mobility of the PVA chains and borate ions in the network, as illustrated in Additional file 1: Fig. S11, Additional files 4 and 5. In general, this decrement in self-healing speed occurs

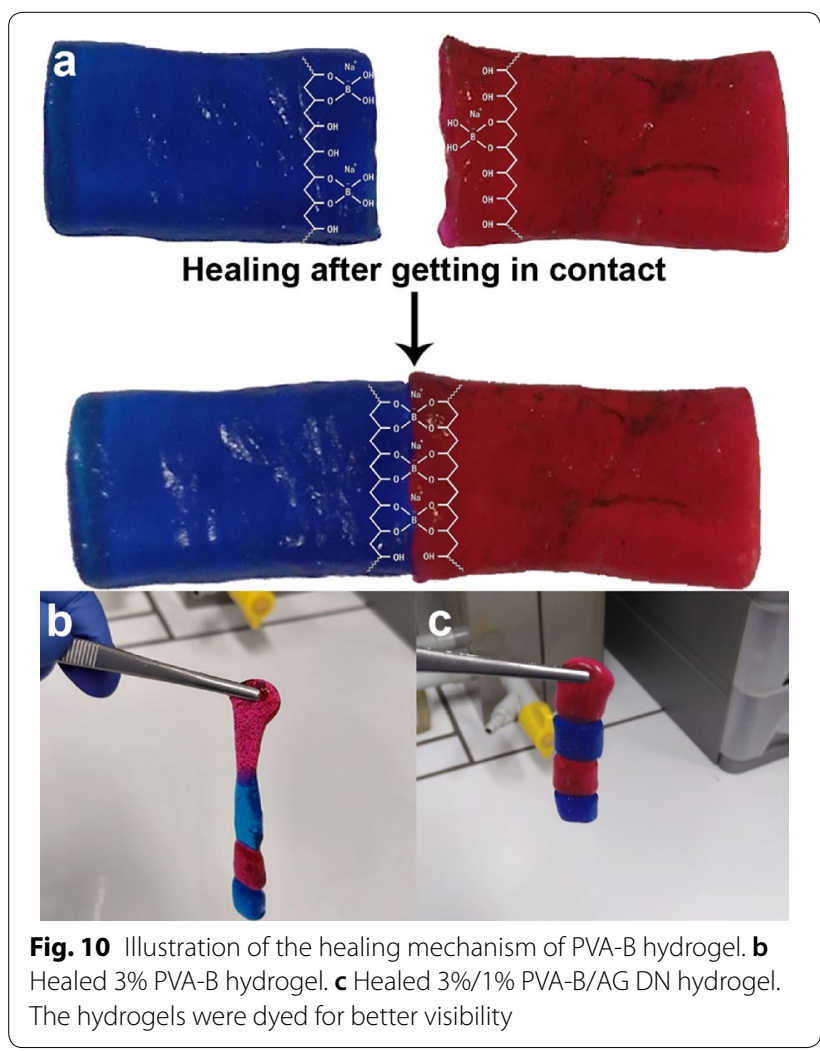

when the gel fraction (PVA and/or AG) is increased. For instance, increasing the concentration of PVA polymer in PVA-B system to $5 \%$ decreases the self-healing speed to approximately $4 \mathrm{~min}$ compared to $2 \mathrm{~min}$ for the $3 \%$ PVA-B hydrogel.

\section{Field tests}

Empirical tests were performed using the PVA-B/AG hydrogel to remove thick soot layers form the ceiling of Osiris sanctuary in the temple of Seti I (19th Dynasty) in Abydos, Egypt. Soot is a common deposit affecting painted surfaces in most of the Egyptian temples and tombs. Its removal is a difficult procedure using traditional cleaning methods. For this reason, a number of cleaning agents that have the ability to remove soot were selected and loaded into the hydrogel. These hydrogel formulations were tested on small spots (diameter of $\mathrm{c}$. $5 \mathrm{~cm}$ ) and the best one was used to treat a large spot (c. $18 \times 10 \mathrm{~cm}^{2}$ ) as a proof of the method. The study also proved the efficiency of the different properties of the hydrogel, such as the shape-stability, self-healing, and the ability to conform to the surface reliefs.

Moreover, the PVA-B/AG hydrogel was used for the treatment of a wall painting in a chapel located in Antwerp-Belgium (nineteenth century). The wall painting was treated with a varnish layer in a previous 
conservation campaign. The deteriorated varnish suffered from substantial darkening. As several traditional cleaning treatments used by conservators lead to damaging of the paint layers underneath the varnish, gel cleaning was proposed as an alternative. After identifying the varnish, several solvent/solvent blends were incorporated into the double network hydrogel and evaluated for their ability to remove the varnish. The best cleaning method was employed to remove the varnish layer safely from the entire wall painting. These two case studies will be described in more detail in forthcoming publications.

\section{Conclusions}

In this study, we investigated the addition of agarose to the PVA-B system and its effect on the chemical structure, liquid phase retention capacity, mechanical properties, rheology behavior, and self-healing properties of the blend. The PVA-B/AG hydrogel features suitable mechanical properties and liquid retention. These features may be ascribed to the formation of hydrogen bonds in the network in addition to the strengthened pores of the hydrogels. In return, this makes PVA-B/AG $\mathrm{DN}$ more suitable for applications on surfaces with high porosity by minimizing the risks of breaking down and leaving residues behind during peeling off. Nevertheless, the PVA-B hydrogels blended with AG exhibit minor syneresis which normally occurs when low polarity solvents or salts (e.g. EDTA) are loaded into the system.

Based on the rheology measurements, AG improves the apparent relaxation time and the shape stability. The PVA-B/AG hydrogels still demonstrate a considerable flexibility, as required for their use on rough surfaces. We showed that PVA-B/AG DN hydrogels exhibit self-healing properties which is considered a beneficial feature that could be useful in some of conservation applications such as the treatment of three-dimensional objects with an irregular surface structure. In conclusion, PVA-B/AG DN hydrogels can serve as a very useful hydrogel and form part of the conservators' cleaning tool-kit. They overcome some of the limitations posed by the unblended PVA-B and AG hydrogels.

It should be noted that this research focused on fully hydrolyzed PVA in order to limit the number of investigated parameters. However, in view of the fact that partially hydrolyzed PVA permits the inclusion of high concentrations of organic solvents, it will be relevant to investigate the properties of blends of different types of partially hydrolyzed PVA (e.g. $80 \%$, $75 \%$, $45 \%$, and $40 \%)$ with agarose. The relative amounts of PVA, borax and AG (and their range) that may lead to hydrogel formulations with enhanced properties such as a higher loading capacity for organic solvents will need to be carefully determined in this more extended parameter space.

\section{Supplementary information}

Supplementary information accompanies this paper at https://doi. org/10.1186/s40494-020-00447-3.

Additional file 1. Hydrogel synthesis, Tables S1-S3, and Figs. S1-S11. Additional file 2. Tensile strength test video (4\% PVA-B hydrogel). Additional file 3. Tensile strength test video (3\%/1\% PVA-B/AG DN hydrogel).

Additional file 4. Self-healing behavior of 3\% PVA-B hydrogel under microscope (video).

Additional file 5. Self-healing behavior of 3\%/1\% PVA-B/AG DN hydrogel under microscope (video).

\section{Abbreviations}

AC: Acetone; AG: Agarose; C25: Ethomeen C25; DN: Double network; EDTA: Ethylenediaminetetraacetic acid; EtOH: Ethanol; FTIR: Fourier transform infrared; PVA-B: Polyvinyl alcohol-borax; Xyl: Xylene.

\section{Acknowledgements}

This article is dedicated to the memory of Prof. Dr. Mohamed Abd El Hady (1949-2019)_-Professor of conservation sciences, Faculty of Archaeology, Cairo University. We express our gratitude to Johan Blom (EMIB research group, University of Antwerp) for his collaboration regarding the rheology measurements. We also thank Jean-Pierre Smet (Department of electromechanics, University of Antwerp) for his valuable assistance in performing the tensile tests. We are grateful to Pieter Van Der Veken and Zunigha De Vos (UAMC research group, University of Antwerp) for helping us in freeze-drying the hydrogels. We thank Karen Leyssens and Bharadwaj Mysore Ramesha (LADCA research group, University of Antwerp) for their valuable assistance in the TGA measurements. We are indebted to Victoria Beltran (AXES research group, University of Antwerp) for her fruitful discussions and comments on the FTIR results.

\section{Authors' contributions}

EA performed all the experiments and measurements and wrote the first draft of the manuscript. HS performed the rheology measurements and wrote the discussion of their data. $\mathrm{KJ}$ and JC contributed to the strategy of the research as well as writing and revising the manuscript. All authors read and approved the final manuscript.

\section{Funding}

Ehab Al-Emam thanks the Egyptian Ministry of Higher Education for funding his Ph.D. scholarship in addition to being grateful to University of Antwerp for additional funding.

Availability of data and materials

All the data are available within the manuscript.

\section{Competing interests}

The authors declare that they have no competing interests.

\section{Author details}

${ }^{1}$ Department of Conservation, Faculty of Archaeology, Sohag University, Sohag 82524, Egypt. ${ }^{2}$ AXES, Faculty of Science, University of Antwerp, Groenenborgerlaan 171, 2020 Antwerp, Belgium. ${ }^{3}$ Nynas NV, Groenenborgerlaan 171, 2020 Antwerp, Belgium. ${ }^{4}$ ARCHES, Faculty of Design Sciences, University of Antwerp, Blindestraat 9, 2000 Antwerp, Belgium.

Received: 23 July 2020 Accepted: 5 October 2020

Published online: 21 October 2020 


\section{References}

1. Angelova LV, Ormsby B, Townsend J, Wolbers R. Gels in the conservation of art. London: Archetype Publications; 2017.

2. Baij L, Hermans J, Ormsby B, Noble P, ledema P, Keune K. A review of solvent action on oil paint. Herit Sci. 2020;8(1):43.

3. El-Gohary M. Experimental tests used for treatment of red weathering crusts in disintegrated granite-Egypt. J Cult Herit. 2009;10(4):471-9.

4. Casoli A, Di Diego Z, Isca C. Cleaning painted surfaces: evaluation of leaching phenomenon induced by solvents applied for the removal of gel residues. Environ Sci Pollut Res. 2014;21(23):13252-63.

5. da Silveira L. A note on the poultice cleaning of feathers using laponite RD gel. Stud Conserv. 1997;42(1):11-6.

6. Wolbers R. Cleaning painted surfaces: aqueous methods. London: Archetype Publications; 2000

7. Angelova LV, Leskes M, Berrie BH, Weiss RG. Selective formation of organo, organo-aqueous, and hydro gel-like materials from partially hydrolysed poly(vinyl acetate)s based on different boron-containing crosslinkers. Soft Matter. 2015;11(25):5060-6.

8. Duncan TT, Weiss RG. Influence of length and structure of aryl boronic acid crosslinkers on organogels with partially hydrolyzed poly(vinyl acetate). Colloid Polym Sci. 2018;296(6):1047-56.

9. Baglioni P, Berti D, Bonini M, Carretti E, Dei L, Fratini E, et al. Micelle, microemulsions, and gels for the conservation of cultural heritage. Adv Colloid Interface Sci. 2014:205:361-71.

10. Duncan TT, Berrie BH, Weiss RG. Soft, peelable organogels from oartially hydrolyzed poly(vinyl acetate) and benzene-1,4-diboronic acid: applications to clean works of art. ACS Appl Mater Interfaces. 2017;9(33):28069-78.

11. Smets A, De Vis K, Ortega-Saez N. A challenging treatment of an 18th century embroidered textile using gel cleaning in combination with decamethylcyclopentasiloxane (D5) silicone solvent barriers. Conservar Património. 2019;31:41-52.

12. Gulotta D, Saviello D, Gherardi F, Toniolo L, Anzani M, Rabbolini A, et al. Setup of a sustainable indoor cleaning methodology for the sculpted stone surfaces of the Duomo of Milan. Herit Sci. 2014;2(1):6.

13. Stockman D. Treatment options for oil stains on paper. The Book and Paper Group Annual. 2007. p. 115-26.

14. Pouliot B, Fair L, Wolbers R. Re-thinking the approach: techniques explored at Winterthur for the stain reduction of ceramics. In: Roemich $\mathrm{H}$, Campagne KVL, editors. Recent advances in glass, stained-glass, and ceramics conservation 2013. Zwolle: SPA Uitgevers; 2013. p. 211-223.

15. Senserrich-Espuñes R, Anzani M, Rabbolini A, Font-Pagès L. Microfragmented agar gels in wall painting conservation: the chapel of Saint Michael in the Royal Monastery of Pedralbes, Barcelona. In: Angelova LV, Ormsby B, Townsend J, Wolbers R, editors. Gels in the conservation of art. London: Archetype Publication; 2017. p. 149-151.

16. Tortajada hernando S, Blanco Domínguez MM. Cleaning plaster surfaces with agar-agar gels: evaluation of the technique. Ge-conservacion. 2013:4:111-26.

17. Hsu H-L, Hsu W-T, Leu J. Effects of environmentally benign solvents in the agarose gel electrolytes on dye-sensitized solar cells. Electrochim Acta. 2011;56(17):5904-9.

18. Miller Z, Whitby G, Garside P. Investigating the ability of phytate gel systems to treat iron gall ink at the British Library. In: Angelova LV, Ormsby B, Townsend J, Wolbers R, editors. Gels in the conservation of art. London: Archetype Publication; 2017. p. 77-81.

19. Cermonesi P, Casoli A. Thermo-reversible rigid agar hydrogels: their properties and action in cleaning. In: Angelova LV, Ormsby B, Townsend J, Wolbers R, editors. Gels in the conservation of art. London: Archetype Publication; 2017. p. 19-28.

20. Bosch-Roig P, Ros JLR, Sancho MPS, Estelles RM, Picazo PR. Biocleaning of wall paintings on uneven surfaces with warm agar gels. In: Angelova LV, Ormsby B, Townsend J, Wolbers R, editors. Gels in the conservation of art. London: Archetype Publication; 2017. p. 119-121.

21. Sousa AMM, Souza HKS, Uknalis J, Liu S-C, Gonçalves MP, Liu L. Electrospinning of agar/PVA aqueous solutions and its relation with rheological properties. Carbohyd Polym. 2015;115:348-55.

22. Koski A, Yim K, Shivkumar S. Effect of molecular weight on fibrous PVA produced by electrospinning. Mater Lett. 2004;58(3):493-7.

23. Mathias BL, Desa JAE, Aswal VK. Reentrant behaviour in polyvinyl alcoholborax hydrogels. Mater Res Express. 2018;5(1):015315.
24. Angelova LV, Terech P, Natali I, Dei L, Carretti E, Weiss RG. Cosolvent gellike materials from partially hydrolyzed poly(vinyl acetate)s and borax. Langmuir. 2011;27(18):11671-82.

25. Angelova LV, Ormsby B, Richardson E. Diffusion of water from a range of conservation treatment gels into paint films studied by unilateral NMR: Part I: acrylic emulsion paint. Microchem J. 2016;124:311-20.

26. Angelova LV, Matarrese C, Fratini E, Weiss RG, Dei L, Carretti E. Chelating agents in aqueous, partially-hydrolyzed, poly(vinyl acetate) dispersions crosslinked with borax Physicochemical characterization and an application. Colloids Surf A Physicochem Eng Aspects. 2018;556:61-71.

27. Angelova LV, Carretti E, Berrie BH, Weiss RG. Poly(vinyl alcohol)-borax 'gels': a flexible cleaning option. In: Angelova LV, Ormsby B, Townsend J, Wolbers R, editors. Gels in the conservation of art. London: Archetype Publication; 2017. p. 231-236.

28. Lyons JG, Geever LM, Nugent MJD, Kennedy JE, Higginbotham CL. Development and characterisation of an agar-polyvinyl alcohol blend hydrogel. J Mech Behav Biomed Mater. 2009;2(5):485-93.

29. Montoro SR, Medeiros SdF, Alves GM. Chapter 10-nanostructured hydrogels. In: Thomas S, Shanks R, Chandrasekharakurup S, editors. Nanostructured polymer blends. Oxford: William Andrew Publishing; 2014. p. 325-355.

30. Kim MS, Kim JW, Yun J, Jeong YR, Jin SW, Lee G, et al. A rationally designed flexible self-healing system with a high performance supercapacitor for powering an integrated multifunctional sensor. Appl Surf Sci. 2020:515:146018.

31. Chen W-P, Hao D-Z, Hao W-J, Guo X-L, Jiang L. Hydrogel with ultrafast selfhealing property both in air and underwater. ACS Appl Mater Interfaces. 2018;10(1):1258-65

32. Domingues JAL, Bonelli N, Giorgi R, Fratini E, Gorel F, Baglioni P. Innovative hydrogels based on semi-interpenetrating p(HEMA)/PVP networks for the cleaning of water-sensitive cultural heritage artifacts. Langmuir. 2013;29(8):2746-55

33. Jin P, Ruan F, Yang X, Liu K, Zou H, Ye L, et al. Assessment of cleaning the corrosion layer of plated bronzes with a complex gel of polyvinyl alcohol and carbomer. Int J Conserv Sci. 2017;8(1):3-14.

34. Bonelli N, Poggi G, Chelazzi D, Giorgi R, Baglioni P. Poly(vinyl alcohol)/ poly(vinyl pyrrolidone) hydrogels for the cleaning of art. J Colloid Interface Sci. 2019;536:339-48.

35. Riedo C, Caldera F, Poli T, Chiantore O. Poly(vinylalcohol)-borate hydrogels with improved features for the cleaning of cultural heritage surfaces. Herit Sci. 2015;3(1):23

36. Al-Emam E, Motawea AG, Janssens K, Caen J. Evaluation of polyvinyl alcohol-borax/agarose (PVA-B/AG) blend hydrogels for removal of deteriorated consolidants from ancient Egyptian wall paintings. Herit Sci. 2019;7(1):22.

37. Snyder LR. Classification of the solvent properties of common liquids. J Chromatogr A. 1974;92(2):223-30.

38. Oliveira RN, Rouzé R, Quilty B, Alves GG, Soares GDA, Thiré RMSM, et al. Mechanical properties and in vitro characterization of polyvinyl alcohol-nano-silver hydrogel wound dressings. Interface Focus. 2014:4(1):20130049.

39. Guirguis OW, Moselhey MTH. Optical study of poly(vinyl alcohol)/ hydroxypropyl methylcellulose blends. J Mater Sci. 2011;46(17):5775-899.

40. Ahad N, Saion E, Gharibshahi E. Structural, thermal, and electrical properties of PVA-sodium salicylate solid composite polymer electrolyte. J Nanomater. 2012;2012:94.

41. Liu C, Bai R. Preparation of chitosan/cellulose acetate blend hollow fibers for adsorptive performance. J Membr Sci. 2005;267(1):68-77.

42. Lim M, Kwon H, Kim D, Seo J, Han H, Khan SB. Highly-enhanced water resistant and oxygen barrier properties of cross-linked poly(vinyl alcohol) hybrid films for packaging applications. Prog Org Coat. 2015;85:68-75.

43. Han J, Wang H, Yue Y, Mei C, Chen J, Huang C, et al. A self-healable and highly flexible supercapacitor integrated by dynamically cross-linked electro-conductive hydrogels based on nanocellulose-templated carbon nanotubes embedded in a viscoelastic polymer network. Carbon. 2019;149:1-18

44. Madera-Santana TJ, Robledo D, Freile-Pelegrín Y. Physicochemical properties of biodegradable polyvinyl alcohol-agar films from the red algae hydropuntia cornea. Mar Biotechnol. 2011;13(4):793-800.

45. Hu Z, Li S, Yang L. Preparation of berbamine loaded chitosan-agarose microspheres and in vitro release study. Polímeros. 2012;22:422-6. 
46. Trivedi TJ, Srivastava DN, Rogers RD, Kumar A. Agarose processing in protic and mixed protic-aprotic ionic liquids: dissolution, regeneration and high conductivity, high strength ionogels. Green Chem 2012;14(10):2831-9.

47. Sabzi M, Samadi N, Abbasi F, Mahdavinia GR, Babaahmadi M. Bioinspired fully physically cross-linked double network hydrogels with a robust, tough and self-healing structure. Mater Sci Eng C. 2017;74:374-81.

48. Chaudhary JP, Kholiya F, Vadodariya N, Budheliya VM, Gogda A, Meena R. Carboxymethylagarose-based multifunctional hydrogel with super stretchable, self-healable having film and fiber forming properties. Arab J Chem. 2020;13(1):1661-8.

49. Rivers S, Umney N. Conservation of furniture. Oxford: Butterworth Heinemann; 2003.

50. Berlangieri C, Andrina E, Matarrese C, Carretti E, Traversi R, Severi M, et al Chelators confined into 80pvac-borax highly viscous dispersions for the removal of gypsum degradation layers. Pure Appl Chem. 2017;89:97.

51. Natali I, Carretti E, Angelova L, Baglioni P, Weiss RG, Dei L. Structural and mechanical properties of "Peelable" organoaqueous dispersions with partially hydrolyzed poly(vinyl acetate)-borate networks: applications to cleaning painted surfaces. Langmuir. 2011;27(21):13226-35.

52. Ferry JD. Viscoelastic properties of polymers. Hoboken: Wiley; 1980.

53. Cai G, Wang J, Qian K, Chen J, Li S, Lee PS. Extremely stretchable strain sensors based on conductive self-healing dynamic cross-links hydrogels for human-motion detection. Adv Sci. 2017;4(2):1600190.

54. Wang Z, Tao F, Pan Q. A self-healable polyvinyl alcohol-based hydrogel electrolyte for smart electrochemical capacitors. J Mater Chem A. 2016;4(45):17732-9.

55. Lu B, Lin F, Jiang X, Cheng J, Lu Q, Song J, et al. One-pot assembly of microfibrillated cellulose reinforced PVA-borax hydrogels with self-healing and pH-responsive properties. ACS Sustain Chem Eng. 2017;5(1):948-56.

\section{Publisher's Note}

Springer Nature remains neutral with regard to jurisdictional claims in published maps and institutional affiliations.

\section{Submit your manuscript to a SpringerOpen ${ }^{\circ}$ journal and benefit from:}

- Convenient online submission

- Rigorous peer review

- Open access: articles freely available online

- High visibility within the field

- Retaining the copyright to your article

Submit your next manuscript at $\boldsymbol{\nabla}$ springeropen.com 